\title{
LA QUIMIOTERAPIA NEOADYUVANTE EN EL TRATAMIENTO DEL CÁNCER DE MAMA LOCALMENTE AVANZADO
}

\author{
Mayer Zaharia1,a, Henry Gómez²,b
}

\section{RESUMEN}

\begin{abstract}
Al inicio del tratamiento del cáncer, se pensaba que este se diseminaba por continuidad, lo cual originó que las primeras estrategias para el manejo de cáncer de mama localmente avanzado fueran altamente agresivas, además de mutilantes. Con los avances en el conocimiento de la biología de la enfermedad, se generaron importantes cambios en el manejo de esta neoplasia. Surgió entonces como alternativa terapéutica la quimioterapia neoadyuvante, que es aquella que se administra previamente al tratamiento quirúrgico. Descrita inicialmente por la escuela de Milán, ha probado ser una mejor alternativa que el tratamiento quirúrgico solo, que la quimioterapia adyuvante (o postquirúrgica) y que la radioterapia sola o en combinación con las técnicas señaladas previamente. Dentro de las ventajas de la quimioterapia neoadyuvante se encuentran el incremento de la tasa de las cirugías conservadoras de mama y la disminución de la tasa de recaída ipsilateral. Facilita además el control y seguimiento de la enfermedad. Sin embargo, la principal desventaja es que no puede modificar algunos factores pronósticos de la enfermedad como la relación tumor mama, enfermedad multicéntrica, las microcalcificaciones dispersas y la coexistencia de procesos médicos que contraindiquen la radioterapia. Actualmente, la quimioterapia neoadyuvante es la base del manejo del cáncer de mama localmente avanzado y está recomendada para garantizar una evolución más conservadora en el manejo de esta enfermedad.
\end{abstract}

Palabras clave: Cáncer de mama; Quimioterapia; Radioterapia; Oncología médica (fuente: DeCS BIREME).

\section{NEOADYUVANT CHEMOTHERAPY IN THE TREATMENT OF LOCALLY ADVANCED BREAST CANCER}

\begin{abstract}
In the first stages of cancer treatment, it was believed that it spreaded by continuity, so the first strategies to treat locally advanced breast cancer were highly aggressive, as well as mutilating. A deeper knowledge of the biology of cancer later resulted in important changes in its treatment, such as the use of neoadyuvant chemotherapy, administered prior to surgical treatment, as an alternative therapy. Initially described by the University of Milan, neoadyuvant chemotherapy has proven to be a better alternative than surgical treatment alone, adjuvant (or postsurgical) chemotherapy and radiotherapy alone or in combination with the previously mentioned techniques. Among its advantages we find an increase in the rate of breast-conserving surgeries and a reduction in the ipsilateral breast tumor recurrence rate. Furthermore, it facilitates breast cancer control and follow-up. On the other hand, its main disadvantage is that it cannot modify some of the disease's prognostic factors, such as the tumor breast relation, multicentric disease, scattered microcalcifications and the coexistence of medical elements which contraindicate radiation therapy. Currently, neoadyuvant chemotherapy is a pillar of the treatment of locally advanced breast cancer and is recommended in order to guarantee a more conservative evolution of the disease.
\end{abstract}

Key words: Breast neoplasms; Chemotherapy; Radioteraphy; Medical oncology (source: MeSH NLM).

\section{INTRODUCCIÓN}

En décadas previas, el manejo del cáncer de mama no metastásico era la resección quirúrgica. La creencia de que la diseminación del cáncer de mama era por vía locorregional generó tratamientos quirúrgicos cada vez más agresivos y mutilantes. Es por ello que en 1950 se definieron los criterios de resecabilidad para el manejo del cáncer de mama. Posteriormente, el conocimiento de la progresión temprana de la enfermedad a través de la vía sanguínea, aunado al hecho de que muchas pacientes acudían a la consulta con lesiones tumorales que no reunían los criterios de resecabilidad, motivó el desarrollo de un tratamiento previo a la cirugía: la neoadyuvancia del cáncer de mama. Descrita en sus inicios por la Escuela de Milán, esta modalidad terapéutica ha evolucionado notablemente en los últimos 25 años, produciendo una revolución en el abordaje clínico, el tratamiento y la investigación del cáncer de mama ${ }^{(1-3)}$

Radio-Oncología. Lima, Perú.

Departamento de Oncología Médica, Instituto Nacional de Enfermedades Neoplásicas. Lima, Perú.

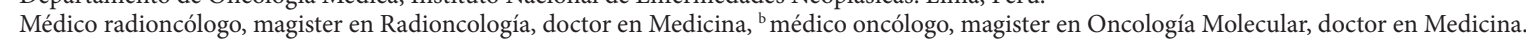
Recibido: 31-01-13 Aprobado: 06-03-13

Citar como: Zaharia M, Gómez H. La quimioterapia neoadyuvante en el tratamiento del cáncer de mama localmente avanzado. Rev Peru Med Exp Salud Publica. 2013;30(1):73-8. 


\section{LA QUIMIOTERAPIA NEOADYUVANTE}

En la década de 1970, investigadores de la Escuela de Milán realizaron los primeros ensayos clínicos sobre terapia neoadyuvante en cáncer de mama, en particular en el cáncer localmente avanzado operable ${ }^{(4-11)}$. Estos primeros ensayos tuvieron como principal limitación que no fueron aleatorizados; sin embargo, demostraron que la quimioterapia neoadyuvante producía tasas de respuestas clínicas elevadas (del 47 al $88 \%$ ), pero con una pobre respuesta patológica completa (del 10 al 30\%). Posteriormente, se realizaron ensayos clínicos en cáncer de mama localmente avanzado inoperable y se obtuvieron tasas de respuestas clínicas similares, con la diferencia que las respuestas patológicas fueron aun más bajas (del 10 al 12\%). En estos estudios destacaron, además, los efectos de la quimioterapia sobre el tumor y los ganglios positivos, observándose que la mitad de los tumores se tornaron operables, una mayor quimiosensibilidad en tumores con receptores hormonales positivos y en los altamente proliferativos; asimismo, se pudo observar que el $91,6 \%$ de los casos con compromiso locorregional aislado en pacientes operadas de inicio, presentó metástasis, mientras que en las pacientes con neoadyuvancia con similares características, solo el $58,1 \%$ presentó metástasis a distancia. ${ }^{(12,13)}$

Sobre la base de los resultados preliminares de la neoadyuvancia en el tratamiento del cáncer de mama localmente avanzado irresecable ${ }^{(4,14-16)}$, y en los estudios que demostraban que la cirugía conservadora y la mastectomía radical eran similares en las pacientes con cáncer de mama temprano, (13,17) surgió la idea de administrar agentes quimioterapéuticos previos a la cirugía (quimioterapia preoperatoria o quimioterapia neoadyuvante) con el objetivo de mejorar la tasa de respuesta en las pacientes, en comparación con la administración del mismo tratamiento tras la intervención quirúrgica (quimioterapia adyuvante).

Surgió entonces el debate para definir cuál de estas dos opciones terapéuticas era la mejor, que como veremos más adelante, terminó por reconocer la eficacia de la quimioterapia neoadyuvante frente a otras alternativas terapéuticas. Lo primero que se debe tener en cuenta es que el manejo del tumor primario, o tumor mamario inicial, ha sido y es controversial. Existía información que sugería que la extirpación del tumor primario podría aumentar el índice de crecimiento de las micrometástasis existentes. En contraste, estudios en modelos animales sugerían que el tratamiento neoadyuvante podría eliminar este riesgo ${ }^{(18-20)}$. Estos datos condujeron al desarrollo de ensayos clínicos que comparaban la quimioterapia prequirúrgica versus la poscirugía ${ }^{(12,13,21-28)}$.

Al término de la década de 1980, una de las primeras consideraciones para evaluar la quimioterapia neoadyuvante en el cáncer de mama era investigar si esta estrategia de tratamiento podía mejorar la supervivencia en pacientes con cáncer de mama localmente avanzado, o en estadios iniciales, es así que algunos de los estudios iniciales señalaban la existencia de una relación entre la respuesta clínica a la quimioterapia neoadyuvante y una mayor tasa de supervivencia libre de recurrencia. Sin embargo, este tipo de diseños no permitían comparar la eficacia de la terapia neoadyuvante en relación a la terapia adyuvante ${ }^{(12,13,21-23)}$. Durante la década de 1990 destacan tres grandes estudios, dos europeos y uno norteamericano, desarrollados con la finalidad de evaluar las diferencias entre administrar la quimioterapia antes y después de la cirugía en cáncer de mama locamente avanzado operable ${ }^{(24-26)}$. La quimioterapia neoadyuvante mostró diferencias significativas en las respuestas clínicas y patológicas tanto a nivel del tumor primario como de los ganglios axilares, en comparación con la terapia adyuvante. Se determinó, además, que la respuesta patológica positiva era un factor pronóstico independiente de sobrevida global ${ }^{(25,26)}$. La eficacia de los tratamientos neoadyuvantes ha sido demostrada por diversos metaanálisis y revisiones sistemáticas, muchos de ellos conducidos por la Universidad de Oxford, los cuales han mostrado un beneficio en las tasas de supervivencia ${ }^{(27-29)}$.

Por todo ello, la quimioterapia neoadyuvante se ha convertido en una estrategia cada vez más utilizada en el tratamiento multidisciplinario del cáncer de mama, y ha sido incluido en diferentes guías de práctica clínica para el manejo de dicho cáncer, como la del National Comprehensive Cancer Network ${ }^{(30)}$. Su uso compromete a todas las especialidades que participan en el manejo de esta enfermedad, constituye, además, un marcador pronóstico de respuesta y un criterio de selección en el tratamiento posterior locorregional y/o sistémico del cáncer de mama localmente avanzado.

\section{DESARROLLO DEL MEJOR ESQUEMA DE QUIMIOTERAPIA}

Tras la primera generación de ensayos clínicos aleatorizados, numerosos estudios han explorado la optimización de diversos regímenes como un camino para mejorar los resultados obtenidos con esta forma de tratamiento, y han sentado las bases racionales biológicas y clínicas para seguir evaluando el papel de la quimioterapia neoadyuvante en el cáncer de mama (27-34). La información inicial sobre quimioterapia neoadyuvante procede de pacientes con carcinoma localmente avanzado de mama tratadas con tres o cuatro ciclos de antraciclinas y de agentes alquilantes. Las tasas de respuesta objetiva, constituida por la suma de respuestas totales y parciales, oscilaban entre el 80 y el $90 \%$, y la tasa de remisiones clínicas completas del 5 al $13 \%{ }^{(26,31,39)}$. 
Hoy en día las drogas más utilizadas son antraciclinas y taxanos, debido a que son más activas en el tratamiento del cáncer de mama avanzado. Tres grandes estudios ${ }^{(32,40,41)}$ han investigado el papel de estos medicamentos en el tratamiento neoadyuvante de pacientes con cáncer de mama en estadios iniciales. En ellos se compararon regímenes de quimioterapia neoadyuvante solo con antraciclinas, solo con antraciclinas y con la combinación de ambos. No se observaron diferencias en cuanto al número de respuestas clínicas ni patológicas.

Los ensayos clínicos con tratamientos secuenciales, en los cuales se administra primero las antraciclinas seguido de los taxanos, han demostrado mayor beneficio, con mejoras en las tasas de respuestas clínicas y patológicas ${ }^{(38,39)}$. Con los años, estos regímenes se han convertido en la práctica más usada en nuestro medio.

Existen diversos estudios de tratamiento neoadyuvante que investigan el papel de la densidad de dosis (esquemas terapéuticos que consideran un menor intervalo de tiempo entre tratamientos que en los esquemas tradicionales) ${ }^{(40,41)}$, los cuales han demostrado un incremento en el número de cirugía conservadoras, siendo significativamente superior en pacientes tratadas con este régimen. Del mismo modo, se ha demostrado con este tratamiento un incremento de la tasa de respuestas completas y un aumento, no significativo, de pacientes con ganglios histológicamente negativos tratadas con esquemas de densidad de dosis.

\section{VENTAJAS DE LA QUIMIOTERAPIA NEOADYUVANTE}

Como se mencionó líneas arriba, los resultados a largo plazo demostraron que la quimioterapia adyuvante (quimioterapia estándar) y la quimioterapia neoadyuvante tienen beneficios similares respecto a la sobrevida global y libre de recaída, por lo que es importante analizar si existen otras ventajas que justifiquen la utilización de la segunda.

La primera de ellas es que la neoadyuvancia permite obtener una tasa más elevada de conservación de la mama, sin un aumento de recidivas tumorales en la mama ipsilateral. Los estudios señalan que los índices de cirugía conservadora de mama eran del $68 \%$ entre las pacientes que recibieron quimioterapia neoadyuvante y del $60 \%$ entre las que recibieron quimioterapia adyuvante ${ }^{(24,39)}$, además en el $23 \%$ de las pacientes que requerían mastectomía al momento del diagnóstico se les pudo realizar una cirugía conservadora tras la quimioterapia neoadyuvante ${ }^{(21)}$.
Otro beneficio demostrado del uso se quimioterapia neoadyuvante, es que la tasa de recaída ipsilateral es del $10,7 \%$ frente al $7,6 \%$ de las pacientes tratadas con quimioterapia adyuvante ${ }^{(42)}$. Ello se debe a que ante la posibilidad de una respuesta parcial a la quimioterapia existirán restos de enfermedad microscópica residual en el tumor primario, y una cirugía conservadora podría dejar enfermedad microscópica residual, en ambos casos aumenta el riesgo de recaída ipsilateral.

La evaluación del tratamiento en el propio tumor o la posibilidad de medir la respuesta tumoral, es un beneficio importante de la quimioterapia neoadyuvante. Al no haber resecado el tumor primario es posible tomar medidas del mismo y de los ganglios axilares, en contraste, cuando la quimioterapia adyuvante se administra después de la resección quirúrgica, la eficacia no puede determinarse hasta que los datos a largo plazo están disponibles, ya que no se cuenta con una muestra tumoral ${ }^{(31-34,40)}$. Este hecho permite tomar actitudes terapéuticas oportunamente. Por ejemplo, en los casos en que las pacientes presenten resistencia a las drogas, determinado por el progreso del tumor durante la quimioterapia neoadyuvante, se debe interrumpir el tratamiento, para pasar a un rescate quirúrgico o radioterapia o segunda línea de quimioterapia. Para la formulación del nuevo esquema terapéutico se debe tener presente la resistencia cruzada (resistencia a los fármacos usados y a otros fármacos a los que el tumor no ha sido expuesto) y evitarla ${ }^{(39)}$.

Por otro lado, la información que nos brinda la relación entre la respuesta tumoral y el pronóstico son diferentes a los que se utilizan en el tratamiento adyuvante. El pronóstico de las pacientes que reciben quimioterapia neoadyuvante depende del grado de enfermedad residual después del tratamiento, en tanto que los factores pronósticos útiles en la quimioterapia adyuvante son el tamaño tumoral y el número de ganglios linfáticos infiltrados. Varios ensayos clínicos han indicado que la ausencia de tumor residual en la mama y en los ganglios linfáticos después de la quimioterapia neoadyuvante, se asocia a un excelente pronóstico a largo plazo ${ }^{(39,43,44)}$.

La terapia neoadyuvante permite establecer la correlación existente entre los marcadores biológicos convencionales obtenidos por punción aspirativa con aguja fina, o biopsia (previa a la administración de este tipo de quimioterapia) y la respuesta tumoral clínica y patológica. Entre estos marcadores se encuentran: el grado histológico, el receptor estrogénico $(R E)$, el receptor de progesterona (RP), el antígeno Ki 67, el oncogen HER-2/neu, entre otros oncogenes. Ello ha permitido diferenciar algunos aspectos. Ahora sabemos que el grado histológico se relaciona con la respuesta ${ }^{(39,42-45)}$, los tumores bien diferenciados 
no suelen alcanzar una respuesta, mientras que casi todas las respuestas ocurren en pacientes con tumores poco diferenciados. Las pacientes cuyo tumor tiene una tasa de proliferación elevada, determinada por el índice mitótico o por Ki-67, presentan una alta tasa de respuesta ${ }^{(45)}$; las pacientes con RE negativos responden más a la quimioterapia neoadyuvante que las pacientes RE positivos (aunque esto puede deberse a que los tumores RE negativos suelen ser de alto grado) (32,44,46,49); las pacientes con tumores HER-2/neu positivos se benefician más de regímenes que contienen antraciclinas ${ }^{(48)}$; finalmente, el grado de apoptosis inducida por el tratamiento puede también predecir la respuesta ${ }^{(49)}$.

La identificación de factores predictivos de respuesta a la quimioterapia puede conducir a tratamientos más eficaces e individualizados, como se demuestra en los datos del MD Anderson Cancer Center (MADCC), en el que se identifica un perfil genético predictivo de respuesta en pacientes que reciben paclitaxel semanal seguido de ciclos FAC (ciclos constituidos por la terapia combinada de 5-fluorouracilo, doxorubicina y cyclophosphamida) ${ }^{(50)}$. Sin embargo, en la actualidad los tratamientos neoadyuvantes están orientados a plataformas genéticas que puedan ser útiles o predictivas para tratamientos específicos.

Dentro de las desventajas del uso de la terapia neoadyuvante se debe mencionar que existen factores que no pueden ser modificados por este tratamiento, tales como la relación tumor-mama, enfermedad multicéntrica, las microcalcificaciones dispersas y la coexistencia de procesos médicos que contraindiquen la radioterapia. EI MDACC definió los criterios de selección y contraindicaciones para una cirugía conservadora tras el tratamiento de quimioterapia neoadyuvante ${ }^{(43)}$. Tampoco se cuenta con información referente a la incidencia de recurrencia local ipsilateral después de mastectomía en pacientes que recibieron quimioterapia neoadyuvante con respuesta patológica completa ${ }^{(51)}$.

\section{QUIMIOTERAPIA NEOADYUVANTE Y RADIOTERAPIA}

Mientras que la quimioterapia se caracteriza por ser un tratamiento sistémico, la radioterapia constituye un tratamiento local y que fue utilizado como terapia única previa a la cirugía en las décadas de los años 70-80. Un ensayo clínico no aleatorizado evaluó la eficacia de la radioterapia en pacientes con cáncer de mama localmente avanzado e inoperable (estadio clínico III [ECIII]), se encontró una tasa de respuesta positiva del $68 \%$, mientras que la supervivencia global a los 5 años en los pacientes con respuesta fue de $32 \%$ en comparación al $5 \%$ alcanzado por los no respondedores; de este estudio se rescata la importancia de la radioterapia en esta enfermedad; aun cuando en él se señala que no existe factores predictivos de respuesta para identificar los pacientes respondedores (6). Posteriormente, se desarrolló un estudio aleatorizado, en pacientes con cáncer de mama EC III, en el cual se evaluaron tres alternativas terapéuticas: radioterapia sola, radioterapia más quimioterapia con el esquema CMF (terapia combinada de ciclofosfamida, metotrexate y 5-fluorouracilo) y radioterapia seguida de cirugía; en otras palabras, se compararon dos tratamientos locorregionales (primer y tercer grupo) y un grupo con tratamiento locorregional seguido de tratamiento sistémico. Se encontró que la supervivencia global a 10 años, estuvo entre 8 a 14\%, sin diferencias estadísticas entre los grupos; sin embargo se encontró que el tercer grupo, en comparación con el segundo, desarrolló el doble de metástasis a distancia (52 y $27 \%$ respectivamente), esta última información favorecía el tratamiento sistémico en la neoadyuvancia ${ }^{(7)}$. Un tercer estudio comparó dos grupos de pacientes con cáncer de mama en quimioterapia con el clásico régimen $\mathrm{CMF}$, en el primer grupo se incluyó dentro del manejo terapéutico radioterapia y cirugía; en tanto que en el segundo grupo solo se incluyó la radioterapia; los resultados de supervivencia a los 10 años no mostraron diferencias entre ambos grupos. Con lo cual se definió el requerimiento del tratamiento sistémico como base del manejo de estos tumores y es recomendando para garantizar una evolución más conservadora en el manejo local de la enfermedad.

Existen indicaciones bien definidas para la radioterapia local en pacientes que reciben quimioterapia neoadyuvante seguida de cirugía conservadora, independientemente del tamaño del tumor primario. Este tratamiento se brinda en el lecho tumoral y puede extenderse a la región infra y supraclavicular y a la cadena mamaria interna ${ }^{(30)}$. Por consenso general en las pacientes con un compromiso ganglionar con cuatro o más ganglios positivos después del tratamiento pre quirúrgico se les indica irradiación de la pared toráxica (dependiendo del tamaño de la lesión primera), aun cuando a este consenso se ha llegado infiriendo los datos provenientes de los estudios de adyuvancia y no de los datos generados en los estudios de neoadyuvancia ${ }^{(47)}$.

\section{CONCLUSIONES}

Diversos estudios han mostrado el beneficio de la quimioterapia y la radioterapia neoadyuvante que, finalmente, concluyeron con su inclusión en diferentes guías clínicas. La disminución de la masa del tumor, uno de los objetivos de la quimioterapia neoadyuvante, permite que los tumores grandes sean operables, 
mientras que la radioterapia disminuye la probabilidad de una recurrencia locorregional. Finalmente, uno de los puntos más importantes, es que permite el estudio de la biología de la respuesta o resistencia al tratamiento, lo que ha conllevado a plantearse si la respuesta del tumor podrá usarse en un futuro para seleccionar el tratamiento y secuencia óptima de tratamiento. Esto podría finalmente conducir a mejorar la supervivencia frente al tratamiento adyuvante estándar. Asimismo, esto ha permitido desarrollar nuevos ensayos destinados a identificar el tratamiento neoadyuvante de acuerdo con las nuevas plataformas genómicas, como el Pam50 (basado en la expresión de 50 genes), que han subdividido el cáncer de mama en varias y diferentes neoplasias, mientras que otros grupos, como los del MD Anderson Cancer Center, desarrollan plataformas genómicas para predecir la respuesta a la quimioterapia neoadyuvante.

\section{REFERENCIAS BIBLIOGRÁFICAS}

1. Fisher B. Laboratory and clinical research in breast cancer -- a personal adventure: the David A. Karnofsky memorial lecture. Cancer Res. 1980;40(11):3863-74.

2. Effects of radiotherapy and surgery in early breast cancer. An overview of the randomized trials. Early Breast Cancer Trialists' Collaborative Group. N Engl J Med. 1995;333(22):1444-55.

3. Polychemotherapy for early breast cancer: an overview of the randomized trials. Early Breast Cancer Trialists' Collaborative Group. Lancet. 1998;352(9132):930-42.

4. Jacquillat C, Weil M, Baillet F, Borel C, Auclerc G, de Maublanc MA, et al. Results of neoadjuvant chemotherapy and radiation therapy in the breast-conserving treatment of 250 patients with all stages of infiltrative breast cancer. Cancer. 1990;66(1):119-29.

5. Bonadonna G, Veronesi U, Brambilla C, Ferrari L, Luini A, Greco M, et al. Primary chemotherapy to avoid mastectomy in tumors with diameters of three centimeters or more. J Natl Cancer Inst. 1990;82(19):1539-45.

6. Zaharia M, Cáceres E. Stage III Breast Cancer. Experience in 15 years at the National Institute of Neoplastic diseases. Acta Cancerológica 1989; XX(2):11-7.

7. Zaharia M, Caceres E, Valdivia S, Moscol A, Pinillos L. Radiotherapy in the management of locally advanced breast cancer. Int J Radiat Oncol Biol Phys. 1987;13(8):1179-82.

8. Bélembaogo E, Feillel V, Chollet P, Curé $\mathrm{H}$, Verrelle P, Kwiatkowski F, et al. Neoadjuvant chemotherapy in 126 operable breast cancers. Eur J Cancer. 1992;28A(4-5):896-900.

9. Smith IE, Jones AL, O'Brien ME, McKinna JA, Sacks N, Baum M. Primary medical (neo-adjuvant) chemotherapy for operable breast cancer. Eur J Cancer. 1993;29A(12):1796-9.
10. Chollet P, Charrier S, Brain E, Curé $\mathrm{H}$, van Praagh I, Feillel V, et al. Clinical and pathological response to primary chemotherapy in operable breast cancer. Eur J Cancer. 1997;33(6):862-6.

11. Gradishar WJ. Docetaxel as neoadjuvant chemotherapy in patients with stage III breast cancer. Oncology (Williston Park). 1997;11(8 Suppl 8):15-8.

12. Mauriac L, MacGrogan G, Avril A, Durand M, Floquet A, Debled M, et al. Neoadjuvant chemotherapy for operable breast carcinoma larger than $3 \mathrm{~cm}$ : a unicentre randomized trial with a 124-month median follow-up. Institut Bergonie Bordeaux Groupe Sein (IBBGS). Ann Oncol. 1999;10(1):47-52.

13. Scholl SM, Fourquet A, Asselain B, Pierga JY, Vilcoq JR, Durand JC, et al. Neoadjuvant versus adjuvant chemotherapy in premenopausal patients with tumours considered too large for breast conserving surgery: preliminary results of a randomised trial: S6. Eur J Cancer. 1994;30A(5):645-52.

14. Smith IE, Walsh G, Jones A, Prendiville J, Johnston S, Gusterson B, et al. High complete remission rates with primary neoadjuvant infusional chemotherapy for large early breast cancer. J Clin Oncol. 1995;13(2):424-9.

15. Anderson ED, Forrest AP, Hawkins RA, Anderson TJ, Leonard RC, Chetty U. Primary systemic therapy for operable breast cancer. $\mathrm{Br} \mathrm{J}$ Cancer. 1991;63(4):561-6.

16. Calais G, Berger C, Descamps P, Chapet S, Reynaud-Bougnoux A, Body G, et al. Conservative treatment feasibility with induction chemotherapy, surgery, and radiotherapy for patients with breast carcinoma larger than $3 \mathrm{~cm}$. Cancer. 1994;74(4):1283-8.

17. Fisher B, Redmond C, Poisson R, Margolese R, Wolmark N, Wickerham
L, et al. Eight-year results of a randomized clinical trial comparing total mastectomy and lumpectomy with or without irradiation in the treatment of breast cancer. $N$ Engl J Med. 1989;320(13):822-8.

18. Gunduz N, Fisher B, Saffer EA. Effect of surgical removal on the growth and kinetics of residual tumor. Cancer Res. 1979;39(10):3861-5.

19. Fisher B, Gunduz N, Saffer EA. Influence of the interval between primary tumor removal and chemotherapy on kinetics and growth of metastases. Cancer Res. 1983;43(4):1488-92.

20. Simpson-Herren L, Sanford $\mathrm{AH}$, Holmquist JP. Effects of surgery on the cell kinetics of residual tumor. Cancer Treat Rep. 1976;60(12):1749-60.

21. Scholl SM, Pierga JY, Asselain B, Beuzeboc P, Dorval T, Garcia-Giralt E, et al. Breast tumour response to primary chemotherapy predicts local and distant control as well as survival. Eur J Cancer. 1995;31A(12):1969-75.

22. Powles TJ, Hickish TF, Makris A, Ashley SE, O’Brien ME, Tidy VA, et al. Randomized trial of chemoendocrine therapy started before or after surgery for treatment of primary breast cancer. J Clin Oncol. 1995;13(3):547-52.

23. Makris A, Powles TJ, Ashley SE, Chang J, Hickish T, Tidy VA, et al. A reduction in the requirements for mastectomy in a randomized trial of neoadjuvant chemoendocrine therapy in primary breast cancer. Ann Oncol. 1998;9(11):1179-84.

24. Fisher B, Brown A, Mamounas E, Wieand S, Robidoux A, Margolese RG, et al. Effect of preoperative chemotherapy on local-regional disease in women with operable breast cancer: findings from National Surgical Adjuvant Breast and Bowel Project B-18. J Clin Oncol. 1997;15(7):2483-93. 
25. Fisher B, Bryant J, Wolmark N, Mamounas E, Brown A, Fisher ER, et al. Effect of preoperative chemotherapy on the outcome of women with operable breast cancer.J Clin Oncol. 1998;16(8):2672-85.

26. Wolmark N, Wang J, Mamounas E, Bryant J, Fisher B. Preoperative chemotherapy in patients with operable breast cancer: nineyear results from National Surgical Adjuvant Breast and Bowel Project B-18. J Natl Cancer Inst Monogr. 2001;30:96-102.

27. von Minckwitz G, Loibl S, Maisch A, Untch M. Lessons from the neoadjuvant setting on how best to choose adjuvant therapies. Breast 2011;20 Suppl 3:S142-5.

28. Mauri D, Pavlidis N, Ioannidis JP. Neoadjuvant versus adjuvant systemic treatment in breast cancer: a meta-analysis. J Natl Cancer Inst. 2005;9(3)7:188-94.

29. Systemic treatment of early breast cancer by hormonal, cytotoxic, or immune therapy. 133 randomised trials involving 31,000 recurrences and 24,000 deaths among 75,000 women. Early Breast Cancer Trialists' Collaborative Group. Lancet. 1992;339(8784):1-15.

30. National Comprehensive Cancer Network (NCCN). Recent Updates to NCCN Clinical Practice Guidelines in Oncology (NCCN Guidelines"). Breast Cancer Version 2.2013 [Internet]. Washington: NCCN; c2013 [citado el 15 de enero del 2013]. Disponible en: http:// www.nccn.org/professionals/physician gls/recently_updated.asp

31. van der Hage JA, van de Velde CJ, Julien JP, Tubiana-Hulin M, Vandervelden C, Duchateau L. Preoperative chemotherapy in primary operable breast cancer: results from the European Organization for Research and Treatment of Cancer trial 10902.J Clin Oncol. 2001;19(22):4224-37.

32. Gianni L, Baselga J, Eiermann W, Porta VG, Semiglazov V, Garcia-Conde J, et al. First report of the European Cooperative Trial in Operable Breast Cancer (ECTO): Effects of primary systemic therapy (PST) on loco-regional disease [abstract]. Proc Am Soc Clin Oncol 2002;21:34a.

33. von Minckwitz G, Costa SD, Raab G, Blohmer JU, Eidtmann H, Hilfrich J, et al. Dose-dense doxorubicin, docetaxel, and granulocyte colony-stimulating factor support with or without tamoxifen as preoperative therapy in patients with operable carcinoma of the breast: a randomized, controlled, open phase IIb study. J Clin Oncol. 2001;19(15):3506-15.

34. Bear HD, Anderson S, Brown A, Smith R, Mamounas EP, Fisher B, et al. The effect on tumor response of adding sequential preoperative docetaxel to preoperative doxorubicin and cyclophosphamide: preliminary results from National Surgical Adjuvant Breast and Bowel Project Protocol B-27. J Clin Oncol. 2003;21(22):4165-74.

35. Smith IC, Heys SD, Hutcheon AW, Miller ID, Payne S, Gilbert FJ, et al. Neoadjuvant chemotherapy in breast cancer: significantly enhanced response with docetaxel. J Clin Oncol. 2002;20(6):1456-66.

36. Green MC, Buzdar AU, Smith T, Ibrahim NK. Weekly (wkly) paclitaxel (P) followed by FAC as primary systemic chemotherapy (PSC) of operable breast cancer improves pathologic complete remission (CRp) rates when compared to every 3-week $(\mathrm{Q}$ $3 \mathrm{wk}) \mathrm{P}$ therapy (tx) followed by FACfinal results of a prospective phase III randomized trial [abstract]. Proc Am Soc Clin Oncol. 2002;21:35a.

37. Untch M, Konecny G, Ditsch N, Sorokina Y, Moebus V, Muck B, et al. Dose-dense sequential epirubicin-paclitaxel as preoperative treatment of breast cancer: results of a randomised AGO study [abstract]. Proc Am Soc Clin Oncol. 2002;21:34a.

38. Fisher B, Mamounas EP. Preoperative chemotherapy: a model for studying the biology and therapy of primary breast cancer. J Clin Oncol.1995;13(3):537-40.

39. Fisher ER, Wang J, Bryant J, Fisher B, Mamounas E, Wolmark N. Pathobiology of preoperative chemotherapy: findings from the National Surgical Adjuvant Breast and Bowel (NSABP) protocol B-18. Cancer. 2002;95(4):681-95

40. Buzdar AU, Singletary SE, Theriault RL, Booser DJ, Valero V, Ibrahim N, et al. Prospective evaluation of paclitaxel versus combination chemotherapy with fluorouracil, doxorubicin, and cyclophosphamide as neoadjuvant therapy in patients with operable breast cancer. J Clin Oncol. 1999;17(11):3412-7.

41. Pouillart P, Fumoleau P, Romieu G, Tubiana-Hulin M, Namer M, Dieras V, et al. Final results of a phase II randomized parallel study of doxorubicin/cyclophosphamide (AC) and doxorubicin/taxol (AT) as neoadjuvant treatment of local-regional breast cancer [abstract]. Proc Am Soc Clin Oncol. 1999; 18:73a.

42. Buchholz TA, Hunt KK, Whitman GJ, Sahin AA, Hortobagyi GN. Neoadjuvant chemotherapy for breast carcinoma: multidisciplinary considerations of benefits and risks. Cancer. 2003;98(6):1150-60.

43. Buzdar AU, Singletary SE, Booser DJ, Frye DK, Wasaff B, Hortobagyi GN. Combined modality treatment of stage III and inflammatory breast cancer. M.D.
Anderson Cancer Center experience. Surg Oncol Clin N Am. 1995;4(4):715-34.

44. Kuerer HM, Newman LA, Smith TL, Ames FC, Hunt KK, Dhingra K, et al. Clinical course of breast cancer patients with complete pathologic primary tumor and axillary lymph node response to doxorubicin-based neoadjuvant chemotherapy. J Clin Oncol. 1999; 17(2):460-9.

45. Wang J, Buchholz TA, Middleton LP, Allred DC, Tucker SL, Kuerer HM, et al. Assessment of histologic features and expression of biomarkers in predicting pathologic response to anthracyclinebased neoadjuvant chemotherapy in patients with breast carcinoma. Cancer. 2002;94(12):3107-14

46. Colleoni M, Gelber S, Coates AS, Castiglione-Gertsch M, Gelber RD, Price $\mathrm{K}$, et al. Influence of endocrine-related factors on response to perioperative chemotherapy for patients with nodenegative breast cancer. J Clin Oncol. 2001;19(21):4141-9.

47. Colleoni M, Minchella I, Mazzarol G, Nolè F, Peruzzotti G, Rocca A, et al. Response to primary chemotherapy in breast cancer patients with tumors not expressing estrogen and progesterone receptors. Ann Oncol. 2000;11(8):1057-9.

48. Thor AD, Berry DA, Budman DR, Muss HB, Kute T, Henderson IC, et al. erbB-2, $\mathrm{p} 53$, and efficacy of adjuvant therapy in lymph node-positive breast cancer. J Natl Cancer Inst. 1998;90(18):1346-60.

49. Buchholz TA, Davis DW, McConkey DJ, Symmans WF, Valero V, Jhingran A, et al. Chemotherapy-induced apoptosis and $\mathrm{Bcl}-2$ levels correlate with breast cancer response to chemotherapy. Cancer J. 2003;9(1):33-41.

50. Pusztai L, Ayers M, Symmans FW, Damokosh A, Hess K, Valero V, et al. Prospective validation of gene expression profiling-based prediction of complete pathologic response to neoadjuvant paclitaxel/FAC chemotherapy in breast cancer [abstract]. Proc Am Soc Clin Oncol. 2003;22:1a.

51. Buchholz TA, Tucker SL, Masullo L, Kuerer HM, Erwin J, Salas J, et al. Predictors of local-regional recurrence after neoadjuvant chemotherapy and mastectomy without radiation. J Clin Oncol. 2002;20(1):17-23.

\footnotetext{
Correspondencia: Mayer Zaharia

Dirección: Paseo de la Republica 3650. Lima 27, Perú.

Teléfono: (511) 422-5520

Correo electrónico:mayerzaharia@hotmail.com
} 